\title{
Some comments on the design of échelle spectrographs using R2 or R4 gratings for precise radial-velocity measurements
}

\author{
John Hearnshaw \\ Dept. of Physics and Astronomy, University of Canterbury, \\ Christchurch, New Zealand \\ Norman Rumsey \\ 21 Malone Rd, Lower Hutt, NZ and Carter Observatory, Wellington, \\ New Zealand \\ Garry Nankivell \\ 43 Holland St., Wainuiomata, Lower Hutt, NZ and Carter Observatory, \\ Wellington, New Zealand
}

\begin{abstract}
.
A new fiber-fed échelle spectrograph (Hercules) is being designed for the 1-m telescope at Mt John University Observatory. The goals are to have a wavelength capability of 380 to $880 \mathrm{~nm}$, covered in a single exposure on a $50-\mathrm{mm}$ square $\mathrm{CCD}$, to have a choice of resolving powers of 35000 or 70000 and to have no moving parts. High precision radialvelocity observations are a major but not the only goal. Designs with both R2 (blaze angle $63.4 \mathrm{deg}$ ) and R4 (blaze angle $76 \mathrm{deg}$ ) échelle gratings are being considered, in either case with a dimension of $408 \mathrm{~mm}$ perpendicular to the grooves.
\end{abstract}

\section{Introduction}

We have designed two new fiber-fed échelle spectrographs to be fed from the Cassegrain focus of the 1-m McLellan telescope at Mt John University Observatory. Both use large échelle gratings of dimension perpendicular to the grooves $L=408 \mathrm{~mm}$; one is an R2 instrument $\left(\theta_{\mathrm{B}}=\arctan 2\right)$ while the other is $\mathrm{R} 4$ $\left(\theta_{\mathrm{B}}=\arctan 4\right)$. We designed two so as to compare their relative merits.

The goals of either design were as follows:

- Resolving power $R=35000$ or 70000 (choice of two discrete values)

- Wavelength range 380 to $880 \mathrm{~nm}$ in continuous coverage for one exposure on a $50-\mathrm{mm}$ square CCD

- High light efficiency

- No moving parts - hence all exposures have the orders falling on the same CCD pixels, giving a high repeatability of dispersion solutions 
- High thermal and mechanical stability, so as to give an excellent precision for radial-velocity measurements

The spectrograph will be known as HERCULES (High Efficiency and Resolution Canterbury University Large Echelle Spectrograph).

\section{Resolving power}

A key parameter for any astronomical spectrograph is the slit- or fiber-limited resolving power, given by

$$
R \propto L \sin \theta_{\mathrm{B}}
$$

where $L$ is the échelle grating size and $\theta_{\mathrm{B}}$ the blaze angle.

Echelle gratings with $\theta_{\mathrm{B}}=63^{\circ} 26^{\prime}$ and $75^{\circ} 58^{\prime}$ are available from the Richardson Grating Laboratory of Spectronics Inc., in each case with $L$ up to $408 \mathrm{~mm}$ perpendicular to the grooves. The values of $\sin \theta_{\mathrm{B}}$ are $0.894(\mathrm{R} 2)$ and 0.970 $(R 4)$. The $R 4\left(\tan \theta_{B}=4\right)$ échelle appears to have a slight advantage (of 8.5 per cent), but $R 4$ gratings introduce several additional complications as discussed below.

\section{Camera focal length}

In either case ( $\mathrm{R} 2$ or $\mathrm{R} 4$ ) we have chosen a grating with 31.6 grooves $/ \mathrm{mm}$. This coarse ruling gives relatively short orders which are well suited to the CCD format.

The order numbers at a given wavelength are similar (ranging from about $n=70$ in the far red to $n=165$ in the near UV) for both échelle gratings, but the higher angular dispersion and angular width of the orders for R4 (by a factor of $\sim 2$ ) mean that a camera of about half the focal length is required, compared to $\mathrm{R} 2$, for the longest red orders to have a length equal to the CCD dimension $(50 \mathrm{~mm})$.

We have designed our instrument to give $R=70000$ in its higher resolving power mode. The camera focal length of any slit-limited spectrograph just satisfying the Nyquist condition of $w^{\prime}=2 \Delta s_{\text {pix }}$ is given by

$$
f_{\text {cam }}=R \cdot \Delta s_{\text {pix }} \cdot \cot \theta_{\mathrm{B}} \cdot\left(1+\tan \theta_{\mathrm{B}} \tan \theta\right)
$$

showing that when $\cot \theta_{\mathrm{B}}=\frac{1}{4}$ (R4) then the camera focal length is half that of the R2 instrument used with the same CCD. Here $w^{\prime}$ is the slit image width on the CCD, and $s_{\text {pix }}$ is the pixel size.

For our spectrographs $f_{\text {cam }}=973 \mathrm{~mm}(\mathrm{R} 2)$ and $459 \mathrm{~mm}(\mathrm{R} 4)$.

\section{Beam size}

The beam size of a spectrograph from the collimator is $A_{\text {coll }}=L \cos \alpha$, provided the échelle grating length $L$ is just filled (though several authors have noted the benefits of overfilling, which is also adopted in Hercules). For our R2 instrument $\theta=3^{\circ}, \alpha=66^{\circ} 26^{\prime}$ and $A_{\text {coll }}=163 \mathrm{~mm}$. The diffracted beam has diameter 
$A_{\text {cam }}=L \cos \beta \simeq 201 \mathrm{~mm}$. These figures compare to $A_{\text {coll }}=A_{\text {cam }}=99 \mathrm{~mm}$ for the R4 collimated and diffracted beams. The R4 instrument operates in quasi-Littrow mode $\left(\theta=0^{\circ}\right.$ - see below). In both spectrographs the collimator is a silvered paraboloid illuminated on axis.

The beam size being about half means that all optical elements are half the size in an R4 spectrograph for a given throughput and resolving power, which in principle would appear to be an attractive cost saving. Since the camera and collimator have half the aperture and focal length, the focal ratios are comparable for these components in both instruments.

\section{Cross dispersion}

Grating cross dispersion is not well suited to a spectrograph in which a large number of orders are to be simultaneously recorded ( $n=64$ to $148-85$ orders - for $\mathrm{R} 2 ; n=70$ to 161 - 92 orders - for R4). In our instruments the fiber diameter is $100 \mu \mathrm{m}$, and the order width (across the orders) on the CCD is the same (about 4 pixels). The mean spacing between order centres is about 588 $\mu \mathrm{m}$ for $\mathrm{R} 2$ and $524 \mu \mathrm{m}$ for $\mathrm{R} 4$, and, as is well known, prism cross dispersion can achieve a more uniform order spacing than a grating. A grism as used by Elodie (Baranne et al., 1996) can achieve more uniformity still (the blue orders are too close for gratings, too separated for prisms).

For our R2 instrument we found that two passes through a Schott BK7 prism of apex angle $50.0^{\circ}$ gives good order separation even in the red. For an R4 instrument, the shorter camera focal length implies that roughly twice as much angular cross dispersion is required, which in turn necessitates either a more dispersive flint glass (our R4 instrument has two LF5 prisms of apex angle $52.5^{\circ}$ ) or three prisms of BK7. Neither is an ideal solution, as LF5 gives too much violet cross dispersion relative to red.

After trying several different alternatives for prism glasses for $\mathrm{R} 4$, which must also have good transmission to $380 \mathrm{~nm}$, no entirely satisfactory solution was obtained if the redmost orders are to be used.

Limiting the prism size is another important consideration. In the R2 spectrograph the BK7 prism is in double pass and close to the échelle. The prism is $280 \mathrm{~mm}$ long and has a base of $220 \mathrm{~mm}$. The wavelength of minimum deviation is $380 \mathrm{~nm}$. The absorption loss in the prism after two passes is 16 per cent at $380 \mathrm{~nm}, 6$ per cent at $420 \mathrm{~nm}$, and less than 3 per cent beyond $660 \mathrm{~nm}$.

In our R4 design both prisms are after the échelle and the white pupil on the échelle is reimaged onto the prisms using two silvered collector mirrors of focal length $3000 \mathrm{~mm}$ and a small fold flat (to reduce the overall length of the instrument - the total length is $3.8 \mathrm{~m}$ ).

\section{Grating illumination}

Two problems with $\mathrm{R} 4$ gratings can be

- facet shadowing, and

- line tilt. 
Facet shadowing occurs when $\alpha>\beta$ and this causes the FWHM of the blaze function to exceed the free spectral range, and a consequent loss in efficiency (Schroeder and Hilliard, 1980). This is so severe for R4 gratings, that quasiLittrow illumination is essential.

Quasi-Littrow illumination $\left(\theta=0^{\circ}\right)$ requires illumination out of the normal plane by an angle $\gamma$, giving line tilt

$$
\phi=\left(2 \tan \theta_{\mathrm{B}}\right) \cdot \gamma=8 \gamma
$$

for R4. In our R4 spectrograph $\gamma$ is set to only $1^{\circ}$, giving $8^{\circ}$ line tilt. The path length to and from the échelle is some $3 \mathrm{~m}$ in order to separate the incident and diffracted beams. Slit rotation (for $R=70000$ ) can partially compensate for line tilt with only one per cent loss in light throughput. For $R=35000$ the circular fiber defines the slit, and the small $8^{\circ}$ line tilt (or shear) can only be compensated for at reduction time.

\section{Camera design}

The simplicity and largely apochromatic properties of the Schmidt camera are attractive for such a large wavelength range. In our R2 design the folded Schmidt incorporates a fold flat mirror with a $100-\mathrm{mm}$ hole to allow access to the $50-\mathrm{mm}$ CCD detector. This inevitably sacrifices about a quarter of the light at many wavelengths. The flat is tilted at $19.0^{\circ}$ so as to allow the CCD dewar to be placed close up behind the hole in the flat.

Folded Schmidt cameras are not ideal because of the light loss entailed. However, they cannot be used at all in the R4 design, because here the hole in the flat would be comparable to the beam size, and all the light would be lost! Refracting cameras are therefore essential, as used in Elodie and in some R2 spectrographs as well (e.g. Libbrecht and Peri (1995) at Palomar). The Libbrecht and Peri camera by Harland Epps has 7 elements, including two of fluorite $\left(\mathrm{CaF}_{2}\right)$ crystals - see also Epps (1998). We have designed three possible refracting cameras for the R4 spectrograph. One is based on the Epps 7-element design and uses two fluorite lenses, and gives the best spot diagrams. We have also studied the possibility of employing less expensive materials, and we have two designs each comprising 10 lens elements with spherical surfaces (three triplets and a BK7 field flattener). One design uses Schott KzFSN4 glass as the central lens in each of three triplets, while another uses FK51 and other glasses. The spot diagrams range from acceptable to good.

The simpler construction and superior aberration performance of the folded Schmidt camera were significant factors favouring building the $\mathrm{R} 2$ design.

\section{Mechanical and thermal mounting}

The spectrograph will be mounted in a sealed tank in a thermally insulated and thermostatted room. The sealed tank will eliminate pressure variations affecting the wavelength. The tank will be evacuated to a few torr so as to eliminate convection currents and refraction effects due to air stratification. The aim is to provide a very stable environment for radial-velocity measurements. 

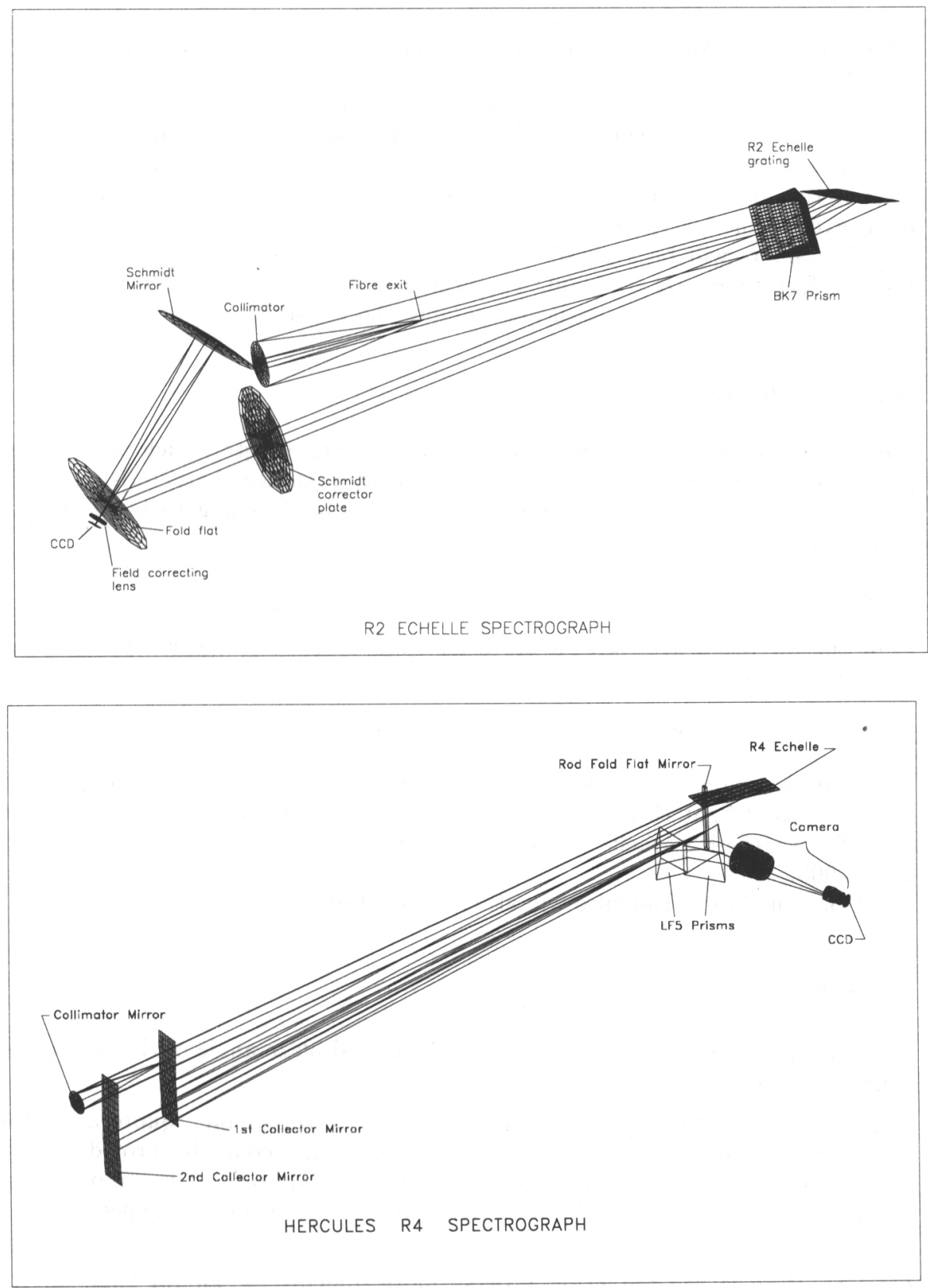

Figure 1. Optical layout of R2 and R4 Hercules spectrographs 


\section{Optical fiber}

About $20 \mathrm{~m}$ of Polymicro $100-\mu \mathrm{m}$ core diameter step index UV-transmitting 'wet' fiber will be used to take the light from the $\mathrm{f} / 8$ Cassegrain focus of the $1-\mathrm{m}$ McLellan telescope to the spectrograph room. The focal ratio will be converted to $\mathrm{f} / 4.3$ at the fiber entrance, and, assuming focal ratio degradation of 10 per cent $(\rho=1.10)$, the beam will emerge within a cone of ratio $f / 3.9$. The fiber core of $100 \mu \mathrm{m}$ corresponds to 4.8 arc s on the sky, so that essentially all the light, even in modest to poor seeing, will be accepted by the fiber.

Two fibers will be available, one of them with a $50-\mu \mathrm{m}$ wide micro-slit at the exit face to give $R=70000$. The other, with no micro-slit, will be used for $R=35000$, with the fiber itself determining the resolution.

\section{Conclusion}

We have designed two spectrographs so as to evaluate factors such as throughput, cost, ease of manufacture and any other performance characteristics. This evaluation is not complete. However, we are probably going to build the R2 design, because of

- a simpler camera with far fewer optical surfaces,

- the far red orders have a better spacing because of the use of the BK7 cross dispersion prism

- the smaller line tilt in the spectra.

The disadvantages of the R2 design are larger heavier optical components and the loss of light through the hole in the fold flat of the Schmidt. Also the cost of the large R2 échelle (about $\$$ US49000) is greater than the R4 at about \$US31 000 .

Construction of Hercules will start in mid-1998.

\section{Discussion}

Latham: How well do you have to know the actual blaze angle of the échelle for your R4 design?

Hearnshaw: The Richardson Grating Lab of Spectronics Inc. quote a typical blaze angle uncertainty of $\pm 0.5^{\circ}$. However a grating could be provided with a measured blaze angle to a precision of $\pm 0.1^{\circ}$ if required. It is important to know the blaze angle, especially so for R4 gratings, before finalizing spectrograph parameters.

Queloz: Why is the reduction process more difficult for an R4 than an R2 grating?

Hearnshaw: First of all, R4 spectrographs require quasi-Littrow illumination $\left(\theta=0^{\circ}\right)$, and this in turn necessitates illumination out of the normal plane $(\gamma \neq$ $\left.0^{\circ}\right)$ in order to separate the incident and diffracted beams. An R4 spectrograph 
with say $\gamma=1^{\circ}$ gives an $8^{\circ}$ line tilt. If this is ignored, it slightly degrades the resolving power, but with a more complex reduction algorithm in which each row of pixels is treated independently, it can be compensated for in the reductions.

Secondly an R4 spectrograph requires an all-refracting camera with a large angular field of view - the field is twice that of an equivalent R2 spectrograph with the same resolving power. That increases the aberrations, including the field distortion. For that reason, the orders may well be curved and the reduction procedure must take this into account. Other aberrations may degrade the resolving power at the edge of the field, complicating radial-velocity reductions. An R2 spectrograph has a camera with a lesser field of view and hence smaller aberrations. We have designed an R2 spectrograph with a folded Schmidt which is apochromatic; the orders are straight and the lines almost untilted.

\section{References}

Baranne, A., Queloz, D., Mayor, M., Adrianzyk, G., Knispel, G., Kohler, D., Lacroix, D., Meunier, J.-P., Rimbaud, G. \& Vin, A. 1996, A\&A Supp. 119,373 .

Epps, H.W. 1998, in Optical astronomical instrumentation, Proc. SPIE Conf. 3355 , paper 64 .

Libbrecht, K.G. \& Peri, M.L. 1995, PASP107, 62.

Schroeder, D.J. \& Hilliard, R.L. 1980, Appl. Optics 19, 2833. 